\title{
DEFICIENCIAS DE NUTRIENTES MINERALES EN SUELOS DEL NORDESTE ARGENTINO. ENSAYOS DE INVERNADERO
}

\author{
Tomei, Carlos E.; Hack, Claudina M. y Porta, Miriam \\ Instituto Agrotécnico "Pedro M. Fuentes Godo", Facultad de Ciencias Agrarias, UNNE \\ tomeice@agr.unne.edu.ar
}

\begin{abstract}
RESUMEN
Esta nota tiene por objeto sistematizar y analizar los resultados obtenidos en distintos trabajos publicados por los autores que han estudiado la existencia de deficiencias nutritivas minerales en suelos del Nordeste Argentino. Los ensayos analizados se ejecutaron en invernadero. Las muestras de suelos se extrajeron de sitios ubicados en las provincias de Misiones, Corrientes, Formosa, Chaco y Santa Fe. Las especies que se emplearon en los ensayos fueron Trifolium repens y Stylosanthes guianensis. A fin de comparar el contenido de $P$ edáfico y la respuesta a la fertilización en cada suelo, se determinó la relación entre rendimiento de MS de los tratamientos con y sin P. Se ratifica que la deficiencia de $P$ es la más importante en los suelos de la subregión húmeda del NEA. Existen otras deficiencias nutricionales minerales que podrían limitar la producción de Trifolium repens y Stylosanthes guianensis, siendo las más frecuentes $\mathrm{Ca}, \mathrm{Mg}, \mathrm{K}, \mathrm{S}$, Mo y $\mathrm{Zn}$. En T. repens el umbral de $P$ edáfico por debajo del cual se pueden esperar respuestas a la fertilización fosfata es de $20 \mathrm{ppm}$. Para establecer el umbral de respuesta al P en $S$. guianensis se deberían estudiar suelos con mayor contenido de $P$ edáfico.
\end{abstract}

Palabras clave: Deficiencias minerales; Trifolium repens; Stylosanthes guianensis

\section{INTRODUCCIÓN}

En la subregión húmeda del Nordeste Argentino (NEA) los suelos son muy heterogéneos, encontrándose diferentes tipos cuya distribución y fertilidad están muy influidas por el origen del material madre. En el nordeste de la región los suelos han evolucionado a partir de roca basáltica, dando lugar a suelos rojos en las lomas y grises en los bajos. Los suelos son pobres en nutrientes y con poder elevado de fijación de fosfatos debido a la presencia de sesquioxidos de hierro y aluminio. Los suelos de la cuenca de los ríos Aguapey, Corriente, Santa Lucía y Paraná derivan de material aluvial antiguo de los que se formaron posteriormente suelos arenosos y arcillo arenosos también pobres en nutrientes.

La fertilidad de los suelos limita la producción y la calidad de los pastizales naturales y de las pasturas cultivadas. La calidad de las pasturas está directamente relacionada a su contenido en nutrientes digestibles, en especial a las sustancias nitrogenadas. Las leguminosas son un componente importante en los sistemas pastoriles debido a su asociación con bacterias fijadoras de N. En los pastizales del NEA la contribución de leguminosas a la producción de materia seca rara vez supera el $5 \%$, aunque existe una población importante en cuanto al número de especies presentes.

La deficiencia mineral más conocida para obtener una adecuada implantación y persistencia de leguminosas de ciclo invernal es la de fósforo, sin embargo en experiencias de campo la fertilización fosfatada no siempre fue suficiente para lograrlo.

Esta nota tiene por objeto sistematizar y analizar los resultados obtenidos en distintos trabajos publicados por los autores que han estudiado la existencia de deficiencias nutritivas minerales en suelos del Nordeste Argentino.

\section{MATERIALES Y MÉTODOS}

Los ensayos analizados se ejecutaron en invernadero, en la Facultad de Ciencias Agrarias, Universidad Nacional del Nordeste, Corrientes, República Argentina.

Las muestras de suelos se extrajeron de sitios ubicados en las provincias de Misiones, Corrientes, Formosa, Chaco y Santa Fe. Las principales características de los suelos que se utilizaron en los ensayos se detallan en la Tabla 1, los análisis químicos fueron realizados en el laboratorio del Instituto Pedro M. Fuentes Godo, Facultad de Ciencias Agrarias, Universidad Nacional del Nordeste.

\section{Determinación de deficiencias}

La determinación de las deficiencias se realizó con el cultivo intensivo de plantas indicadoras en macetas, los resultados analizados fueron obtenidos 
Tabla 1.- Clasificación y propiedades físico químicas de los suelos.

\begin{tabular}{|c|c|c|c|c|c|c|c|}
\hline \multirow{2}{*}{ Suelo } & \multirow{2}{*}{ Clasificación } & \multirow{2}{*}{$\mathrm{pH}$} & C.O. & $\mathrm{P}$ & $\mathbf{K}$ & $\mathrm{Ca}$ & $\mathbf{M g}$ \\
\hline & & & $\%$ & $\mathrm{ppm}$ & \multicolumn{3}{|c|}{$\mathrm{cmol} / 100 \mathrm{~g}$} \\
\hline \multicolumn{8}{|l|}{$\overline{\text { Corrientes }}$} \\
\hline Sombrero & Argiudol acuico & 5.7 & 1.43 & 5 & 0,07 & 8,9 & 0.6 \\
\hline San Luis & Argiudol Acuico & 6,0 & 1,0 & 4,0 & 0,05 & 4,3 & 0,82 \\
\hline Mercedes & Argiudol Acuico & 5,8 & 1,97 & 5 & 0,07 & 9,2 & 0,1 \\
\hline Yofre & Argiudol Vértico & 5,9 & 1,86 & 8 & 0.098 & 6,9 & 1,0 \\
\hline Ituzaingó & Udifluvent Acuico & 6,8 & 0.7 & 3 & 0,075 & 2,4 & 0,2 \\
\hline Virasoro & Kandihumult Típico & 5,1 & 3,02 & 5,0 & 0,14 & 5,7 & 1,2 \\
\hline Perugorria & Argiudol Típico & 6,6 & 2,9 & 7 & 0,25 & 18 & 6,8 \\
\hline Paso Martínez & Argiudol Acuico & 5,9 & 0,9 & 6 & 0,14 & 3,1 & 0,9 \\
\hline Loreto & Udipsament alfico & 5,7 & 0,4 & 5 & 0,10 & 0,2 & 1,8 \\
\hline Río Corriente & Argiacuol Típico & 5,9 & 1,72 & 9 & 0,074 & 5,2 & 1,9 \\
\hline Curuzú & Argiudol Vértico & 5.5 & 2,0 & 4,0 & 0,128 & 4,0 & 0,54 \\
\hline Brugne & Argiudol Acuico & 5,3 & 1,53 & 5 & 0.69 & 2,5 & 1 \\
\hline Miriñay & Ocracualf Aérico & 5,2 & 2,7 & 6,0 & 0,146 & 4,1 & 2,0 \\
\hline \multicolumn{8}{|l|}{ Misiones } \\
\hline Jardín América & Haplustol Entico & 5,9 & 4,83 & 2,42 & 0,29 & 20,4 & 2,5 \\
\hline \multicolumn{8}{|l|}{ Santa Fe } \\
\hline Los Laureles & Albacualf Tipico & 6,5 & 0,93 & 4 & 0,60 & 22 & 0.5 \\
\hline Tartagal & Albacualf Típico & 6,1 & 2,07 & 87 & 1,34 & 14,4 & 4,8 \\
\hline Moussy & Argiudol Acuico & 6,0 & 2,74 & 6,0 & 0,54 & 12,5 & 10,8 \\
\hline Avellaneda & Argialbol Típico & 6,0 & 1,19 & 10 & 0,24 & 7,0 & 6,6 \\
\hline \multicolumn{8}{|l|}{ Chaco } \\
\hline Tirol & Haplacuept Vértico & 7,3 & 2,58 & 10 & 0,34 & 43,3 & 8.3 \\
\hline Vedia & Argiustol Acuico & 6.0 & 1.6 & 7 & 0,115 & 4,6 & 1,9 \\
\hline \multicolumn{8}{|l|}{ Formosa } \\
\hline Mbiguá & Haplustol Oxico & 6,0 & 1,0 & 22.0 & 0,36 & 4.0 & 0.46 \\
\hline
\end{tabular}

Tabla 2.- Indice de Respuesta al $\mathrm{P}$

\begin{tabular}{ll}
\hline & MS P/MS T \\
\hline $1,00-1,25$ & 0 \\
$1,26-1,50$ & 1 \\
$1,51-1,75$ & 2 \\
$1,76-2,00$ & 3 \\
$2,01-2,25$ & 4 \\
$2,26-2,50$ & 5 \\
$2,51-2,75$ & 6 \\
$2,76-3,00$ & 7 \\
$3,01-3,25$ & 8 \\
$3,26-3,50$ & 9 \\
$3,51 \mathrm{a}+$ & 10 \\
\hline
\end{tabular}

entre 1995 y 2005 . Las macetas fueron de $0,5 \mathrm{~kg}$ de capacidad y se llenaron hasta $2 \mathrm{~cm}$ del borde, se agregaron los nutrientes y se sembraron con 50 semillas. Como plantas indicadoras se utilizaron Trifolium repens y Stylosanthes guianensis, la primera en siembras de Marzo, la segunda de Septiembre. Se estudió el efecto del agregado de $\mathrm{P}, \mathrm{K}$, $\mathrm{Ca}, \mathrm{Mg}, \mathrm{S}, \mathrm{Mo} \mathrm{Zn}, \mathrm{Cu}$ y $\mathrm{B}$ con una técnica aditiva. Los nutrientes se agregaron como producto puro en solución, excepto $\mathrm{Ca}$ y $\mathrm{Mg}$ que se aplicaron como carbonatos en polvo (Tamiz $\mathrm{N}^{\circ} 1$ ). Se usó un diseño estadístico en bloques al azar de diez tratamientos con tres repeticiones. La variable de respuesta analizada fue la producción de materia seca (MS). Las plantas fueron cortadas a $2 \mathrm{~cm}$ de altura, se secaron en estufa de tiro forzado a $60^{\circ}$ $\mathrm{C}$, hasta peso constante. Los datos de MS fueron sometidos al análisis de variancia y las diferencias entre medias se probaron con la prueba de Tukey ( $p>5,00 \%)$.

A fin de comparar el contenido de $\mathrm{P}$ del suelo y la respuesta a la fertilización con $P$ en cada suelo, se determinó la relación entre la producción de MS del tratamiento con $\mathrm{P}$ y la producción de MS del testigo. Con los resultados se estableció una escala de indice respuesta (IR) a la fertilización (Tabla 2). Se realizó el análisis de regresión y se obtuvo la ecuación de respuesta correspondiente para $T$. repens y $S$. guianensis.

\section{RESULTADOS Y DISCUSIÓN \\ Deficiencias primarias:}

Para $T$. repens la mayoria de los suelos estudiados mostraron deficiencias primarias en uno o más nutrientes además del P (Tabla 3). Se explican así los resultados desfavorables en los intentos de cultivo de esta leguminosa forrajera en suelos del Nordeste Argentino al aplicar solamente fertilizantes fosfatados.

Con $S$. guianensis ocurrió algo similar a lo ob- 
Tabla 3.- Deficiencias encontradas para $T$. repens y $S$. guianensis

\begin{tabular}{|c|c|c|c|c|c|}
\hline \multirow{3}{*}{ Localidad } & \multirow{3}{*}{ Tipo de suelo } & \multicolumn{2}{|c|}{ T. repens } & \multicolumn{2}{|c|}{ S. guianensis } \\
\hline & & \multirow{2}{*}{$\begin{array}{c}\text { Def. } \\
\text { Primaria }\end{array}$} & \multirow{2}{*}{$\begin{array}{l}\text { Def. } \\
\text { Sec. }\end{array}$} & \multirow{2}{*}{$\begin{array}{c}\text { Def. } \\
\text { Primaria }\end{array}$} & \multirow{3}{*}{$\begin{array}{l}\text { Def. } \\
\text { Sec. }\end{array}$} \\
\hline & & & & & \\
\hline Sombrero, Corrientes & Argiudol Acuico & $\mathrm{P}$ & - & $\mathbf{P}$ & \\
\hline San Luis, Corrientes & Argiudol Acuico & $\mathrm{PCaS}$ & - & $\mathrm{PZn}$ & $\mathrm{Mg} \mathrm{Mo}$ \\
\hline Mercedes, Corrientes & Argiudol Acuico & P K & - & P K & - \\
\hline Paso Martinez; Corrientes & Argiudol Acuico & * & * & P Ca K & - \\
\hline Brugne, Corrientes & Argiudol Acuico & $*$ & * & P B & - \\
\hline Yofre, Corrientes & Argiudol Vértico & P K & $\mathrm{Mg}$ & $\mathrm{PCa}$ & $\mathrm{Mg} \mathrm{Mo}$ \\
\hline Curuzú, Corrientes & Argiudol Vértico & $\mathbf{P}$ & Mo & $\mathrm{PCa}$ & $\mathrm{Mg}$ \\
\hline Perugorria, Corrientes & Argiudol Típico & $\mathrm{PCu}$ & Mo Mg & $\mathrm{P}$ & $\mathrm{S}$ \\
\hline Rio Corriente, Corrientes & Argiacuol Típico & $\mathrm{P}$ & Mo & $\mathrm{P}$ & - \\
\hline Ituzaingó, Corrientes & Udifluvent Acuico & $\mathbf{P}$ & $\mathrm{Ca}$ & $\mathrm{P}$ & Mo \\
\hline Loreto, Corrientes & Udipsament Alfico & $*$ & $*$ & P Mg S & - \\
\hline Miriñay, Corrientes & Ocracualf Aérico & $*$ & * & - & - \\
\hline Virasoro, Corrientes & Kandihumult Típico & * & * & P Mg S & - \\
\hline Jardin América, Misiones & Haplustol Entico & P Mo & - & $\mathbf{P}$ & Mo \\
\hline Los Laureles, Santa Fe & Albacualf Tipico & $\mathrm{PZn}$ & $\mathrm{Ca} \mathrm{S}$ & $*$ & $*$ \\
\hline Tartagal, Santa Fe & Albacualf Típico & $\mathrm{Zn}$ & $\mathbf{P}$ & * & $*$ \\
\hline Avellaneda, Santa Fe & Argialbol Típico & $\mathrm{PCa}$ & - & $*$ & $*$ \\
\hline Moussy, Santa Fe & Argiudol Acuico & $\mathrm{P} \mathrm{Ca} \mathrm{Mg}$ & - & $*$ & $*$ \\
\hline Tirol, Chaco & Haplacuept Vértico & $\mathrm{P} \mathrm{Mg}$ Mo & - & * & $*$ \\
\hline Vedia, Chaco & Argiustol Acuico & P S Mo & - & P Mo & - \\
\hline Mbiguá, Formosa & Haplustol Oxico & - & - & $*$ & $*$ \\
\hline
\end{tabular}

Ref.: Signo - = no hay deficiencia; signo $*$ = no se cultivó la planta indicadora

Tabla 4.- Frecuencia de las deficiencias primarias.

\begin{tabular}{|c|c|c|c|c|c|c|c|c|c|c|c|c|c|c|}
\hline \multirow{2}{*}{$\begin{array}{c}\text { Planta } \\
\text { indicadora }\end{array}$} & \multirow{2}{*}{\multicolumn{2}{|c|}{$\begin{array}{l}\text { Total de } \\
\text { suelos }\end{array}$}} & \multicolumn{12}{|c|}{ Deficiencias primarias } \\
\hline & & & $\mathrm{P}$ & & K & & $\mathrm{Ca}$ & & $\mathrm{Mg}$ & $S$ & Mo & $\mathrm{Zn}$ & $\mathrm{Cu}$ & B \\
\hline T.repens & 16 & & 14 & 2 & & 3 & & 2 & & 2 & 3 & 2 & 1 & 0 \\
\hline S. guianensis & 15 & & 14 & 2 & & 3 & & 2 & & 2 & 1 & 2 & 0 & 1 \\
\hline
\end{tabular}

Tabla 5.- Frecuencia de las deficiencias secundarias.

\begin{tabular}{|c|c|c|c|c|c|c|c|c|c|c|c|c|}
\hline \multirow{2}{*}{ Planta indicadora } & \multirow{2}{*}{\multicolumn{2}{|c|}{ Total suelos }} & \multicolumn{10}{|c|}{ Deficiencias secundarias } \\
\hline & & & $\mathbf{P}$ & & $\mathrm{K}$ & $\mathrm{Ca}$ & & $\mathrm{Mg}$ & Mo & $\mathrm{Zn}$ & $\mathrm{Cu}$ & B \\
\hline T.repens & 16 & & 1 & 0 & & 2 & 2 & 1 & 3 & 0 & 0 & 0 \\
\hline S. guianensis & 15 & & 0 & 0 & & 0 & 3 & 1 & 4 & 0 & 0 & 0 \\
\hline
\end{tabular}

servado con $T$. repens, ya que la deficiencia de $P$ es acompañada generalmente por deficiencias en otros nutrientes. La única deficiencia en la que coinciden en todos los casos estas dos especies para un mismo suelo fue en la de P. Las deficiencias encontradas no estuvieron asociadas a tipos particulares de suelos.

\section{Frecuencia de las deficiencias primarias:}

De acuerdo a lo esperado, la deficiencia primaria más frecuente fue la de $\mathrm{P}$, tanto para $T$. repens como para $S$. guianensis (Tabla 4). Le siguieron al $P$ como deficiencias primarias Ca y Mo para trébol blanco y con menor frecuencia $\mathrm{K}, \mathrm{Mg}, \mathrm{S}$ y $\mathrm{Zn}$. En un solo suelo apareció el $\mathrm{Cu}$ como deficiencia. $\mathrm{Si}$ bien la deficiencia de $P$ fue la más frecuente, existen otras deficiencias que pueden hacer fracasar intentos de cultivo si no se agregan esos nutrientes.
Es el caso del Zn en el norte de la Provincia de Santa Fé o de Corrientes y del $\mathrm{S}$ en suelos del este del Chaco. S. guianensis mostró la misma distribución de frecuencias en las deficiencias, excepto en Mo, Cu y B.

\section{Deficiencias secundarias:}

Las deficiencias secundarias, cuyo rol a largo plazo falta dilucidar, fueron menos frecuentes y se remiten a uno o dos nutrientes.

Para el trébol blanco las deficiencias secundarias más frecuentes fueron $\mathrm{Ca}, \mathrm{Mg}$, Mo y $\mathrm{S}$. El P se mostró como deficiencia secundaria solo en un suelo con alto contenido de este nutriente. Para $S$. guianensis las deficiencias secundarias encontradas fueron $\mathrm{Mg}$, Mo y S (Tabla 5).

Las deficiencias secundarias probablemente indiquen problemas de disponibilidad de esos nu- 
Tabla 6.- Contenido de P (ppm) e Indice de Respuesta en T.repens

\begin{tabular}{lll}
\hline \multicolumn{1}{c}{ Suelo } & & P ppm \\
\hline Yofre & 8 & 6 \\
Mercedes & 5 & 10 \\
Curuzú & 4 & 2 \\
Ituzaingó & 3 & 2 \\
San Luis & 4 & 5 \\
Sombrero & 5 & 10 \\
Perugorria & 7 & 6 \\
Jardín América & 2,4 & 3 \\
Los Laureles & 4 & 3 \\
Tartagal & 87 & 0 \\
Moussy & 6 & 1 \\
Avellaneda & 10 & 2 \\
Tirol & 10 & 3 \\
Vedia & 7 & 2 \\
Río Corriente & 9 & 2 \\
Mbiguá & 22 & 0 \\
\hline
\end{tabular}

Tabla 7.- Contenido de $\mathrm{P}$ (ppm) e Indice de Respuesta en $S$. guianensis

\begin{tabular}{llll}
\hline \multicolumn{1}{c}{ Suelo } & & $\begin{array}{c}\text { P } \\
\text { (ppm) }\end{array}$ & IR \\
\hline Yofre & 8 & 7 \\
Mercedes & 5 & 2 \\
Curuzú & 4 & 1 \\
Ituzaingó & 3 & 2 \\
San Luis & 4 & 1 \\
Perugorria & 7 & 9 \\
Sombrero & 5 & 7 \\
Virasoro & 5 & 4 \\
Loreto & 5 & 3 \\
Río Corriente & 9 & 2 \\
Paso Martínez & 6 & 10 \\
Jardin América & 2,4 & 5 \\
Vedia & 7 & 6 \\
Brugne & 5 & 10 \\
Miriñay & 6 & 0 \\
\hline
\end{tabular}

trientes a largo plazo, es una cuestión que se debe dilucidar con experimentos de campo o de invernadero, dentro de las limitaciones de esta ultima alternativa.

\section{Respuesta a la fertilización fosfatada:}

Utilizando los Indices de Respuesta (IR) definidos anteriormente (Tabla 2), para cada suelo se calculó la relación de estos con los contenidos de P (Tabla 6).

En $T$. repens se observó respuesta a la fertilización con $P$ cuando el contenido de este nutriente en el sue-

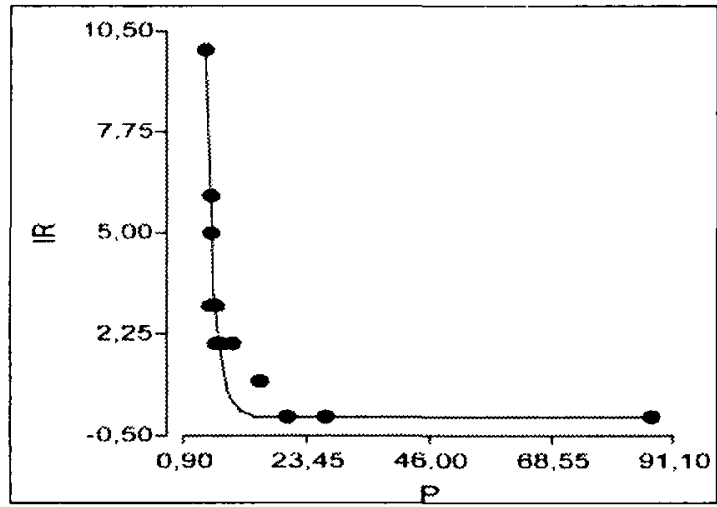

Figura 1. Relación respuesta al $P$ y contenido de $P$ en el suelo, Trepens.

lo estaba por debajo de $20 \mathrm{ppm}$, por encima de este nivel crítico no se produjeron respuestas (Fig. 1).

Este comportamiento es explicado por un modelo exponencial del tipo: $I R=235,01 * \exp (-0,64 \mathrm{P})$. Este resultado coincide con Melgar (1997), quién reporta que por encima de contenidos de $15 \mathrm{ppm}$ de $\mathrm{P}$ edáfico disponible la probabilidad de respuesta es baja.

Si bien en la mayoria de los suelos junto a la deficiencia de fósforo se encontraron otras deficiencias acompañantes, la relación entre el contenido de $P$ del suelo y la respuesta del $T$. repens a la fertilización fosfatada tuvo buen ajuste.

En el caso del $S$. guianensis no hubo una relación tan estrecha entre respuesta al agregado de P y contenido edáfico de este nutriente como en trébol blanco. Esto puede deberse en parte a que, todos los suelos estudiados con esta planta indicadora contenían como máximo $10 \mathrm{ppm}$ de $\mathrm{P}$ (Tabla 1) y no se estudiaron suelos con tenores superiores.

En $S$. guianensis hubo respuesta al agregado de $\mathrm{P}$ en prácticamente todos los suelos, a excepción de uno (tabla 4), aunque no pudo establecerse ninguna relación con el $\mathrm{P}$ edáfico (Tabla 7). Probablemente este comportamiento se deba al efecto de las otras deficiencias encontradas, lo que indica que $S$. guianensis seria más sensible a estas situaciones que el trébol blanco.

\section{CONCLUSIONES}

Se ratifica que la deficiencia de $\mathrm{P}$ es la más importante en los suelos de la subregión húmeda del NEA.

Existen otras deficiencias nutricionales minerales que podrían limitar la producción de Trifolium repens y Stylosanthes guianensis, siendo las más frecuentes $\mathrm{Ca}, \mathrm{Mg}, \mathrm{K}, \mathrm{S}$, Mo y $\mathrm{Zn}$.

En $T$. repens el umbral de $\mathrm{P}$ edáfico por debajo del cual se pueden esperar respuestas a la fertilización fosfata es de $20 \mathrm{ppm}$.

Para establecer el umbral de respuesta al $\mathrm{P}$ en $S$. guianensis se deberían estudiar suelos con mayor contenido de $\mathrm{P}$ edáfico. 


\section{BIBLIOGRAFÍA}

Tomei, C.E.; Castelán, M.E.; Poletti, M.M. y Slukwa, M.A. 1995. Respuesta del Trifolium repens al agregado de $\mathrm{P}, \mathrm{K}, \mathrm{Ca}, \mathrm{Mg} \mathrm{S}$ en ocho suelos del Nordeste Argentino. Revista de la Facultad de Agronomía, La Plata Tomo 71 (2): $173-178$.

Tomei, C.E.; Regonat, P.; Tomei, C.E.(h); Castelán, M.E. y Arce, G. 1996. Ensayos exploratorios en macetas sobre fertilidad de suelos del Chaco Oriental Argentino. Agrotecnia 2: $1-6$.

Tomei, C. E.; Castelan, M. E.; Ciotti, E.M.; Benitez, J.A. y Huguet, H. H. 1999. Deficiencias nu- tritivas en Stylosanthes guianensis CIAT 184 en suelos de Corrientes, Argentina. Agrotecnia 5: $16-20$,

Tomei, C. E., Ciotti, E. M., Castelán, M. E., Hack, C. M. y Pomarada, L. 2001. Determinación de deficiencias minerales y su corrección para Trifolium repens $\mathrm{L}$. en un argiustol acuico. Agrotecnia $\mathrm{N}^{\circ}$ 7: 2-8.

Tomei, C.E.; Britos, N.; Castelán, M.E.; Ciotti, E.M. y Hack, C.M. 2002. Deficiencias minerales y su corrección para Stylosanthes guianensis en un Argiudol Vértico. Agrotecnia $N^{\circ}$ 8: 22-23. 